\title{
Crianças com fissura labiopalatina e baixo peso ao nascimento em testes auditivos centrais
}

\section{Children showing labial palatine fissures and a low weight at birth in central hearing tests}

\author{
Letícia Maria Martins Araújo', José Roberto Pereira Lauris², Mariza Ribeiro Feniman³.
}

1) Fonoaudióloga. Discente do Curso de Especialização em Audiologia do Instituto de comunicação e Audição (ICA) - Bauru/SP

2) Livre-Docência. Professor Associado do Departamento de Fonoaudiologia da Faculdade de Odontologia de Bauru-USP

3) Professora Titular do Departamento de Fonoaudiologia da FOB-USP. Chefe de Departamento de Fonoaudiologia-FOB-USP

Instituição: Faculdade de Odontologia de Bauru da Universidade de São Paulo - FOB - USP.

Bauru / SP - Brasil.

Endereço para correspondência: Mariza Ribeiro Feniman - Departamento de Fonoaudiologia da Faculdade de Odontologia de Bauru (FOB-USP) -Alameda Octávio Pinheiro Brisolla 9-75 - Bauru / SP - Brasil - CEP: 17012-901 - Telefone: (+55 14) 3235-8533 - Fax: (+55 14) 3223-4679 - E-mail: feniman@usp.br

Artigo recebido em 11 de Março de 2011. Artigo aprovado em 15 de Maio de 2011.

\section{RESUMO}

Introdução: Alterações neurológicas ou sensoriais, entre elas as alterações auditivas periféricas e/ou centrais, são enfatizadas na população com baixo peso ao nascimento (BP), assim como, na presença de malformação craniofacial, tal como a fissura labiopalatina (FLP).

Objetivo: Verificar e comparar o resultado de testes de processamento auditivo, Teste Dicótico de Dígitos (TDD) e Teste de Fusão Auditiva-Revisado (AFT-R), de crianças com FLP com e BP ao nascimento, com o de crianças sem FLP e nascida com peso normal.

Método: Estudo retrospectivo e comparativo de 73 prontuários, dos quais foram verificados o sexo, peso ao nascimento, presença/ausência de FLP e o resultado de TDD e AFT-R. Foram constituídos três grupos de acordo com a análise do peso ao nascimento e presença ou ausência de FLP.

Resultados: Para o TDD a Análise de Covariância não mostrou diferença entre os grupos e sexos, porém a co-variável idade mostrou relação estatisticamente significante.Para o AFT$\mathrm{R}$ não mostrou diferença entre os grupos, sexos e idades.

Conclusão: Crianças com FLP e BP, embora sem significância estatística, apresentam maiores alterações nos testes de processamento auditivo utilizados ao comparar com crianças com FLP isolada e com crianças sem esta malformação craniofacial e sem BP.Ressalta-se ainda que o aumento da idade melhorou o desempenho no TDD.

Palavras-chave: percepção auditiva, fissura palatina testes auditivos.

\section{SUMMARY}

Introduction: Neurological or sensorial alterations, some of which are peripheral and/or central hearing alterations, are focused on both the people showing a low weight at birth (LW) and the presence of craniofacial malformation, such as labial palatine fissure (LPF).

Objective: Check and compare the results of hearing processing tests, Dichotic Listening Test (DLT) and Auditory Fusion TestRevised (AFT-R) in LPF and LW children with those in children born with a normal weight and showing no LPF.

Methods: Retrospective comparative study with 73 records, in which sex, weight at birth, LPF presence/absence and the DLT and AFT-R results were verified. Three groups were formed based on the analysis of weight at birth and FLP absence/ presence.

Results: For DLT, the Covariance Analysis did not present any difference between the groups and sexes; however the age covariant showed a statistically significant relation. Fort AFT$\mathrm{R}$, there was no difference between groups, sexes and ages. Conclusion: LPF AND LW children, however statistically insignificant, showed bigger modifications in the hearing processing tests in comparison with isolated LPF children and with children having neither this craniofacial malformation nor LW. It must as well be emphasized that an increase in age enhanced DDT performance.

Keywords: auditory recognition; palatine fissure; hearing tests 


\section{INTRODUÇÃO}

Alterações neurológicas ou sensoriais, entre elas as alterações auditivas periféricas e/ou centrais, são enfatizadas na população com baixo peso ao nascimento (1-5), assim como na presença de malformação craniofacial, tal como a fissura labiopalatina (6-12).

O transtorno de processamento auditivo é uma alteração auditiva central, refere às dificuldades no processamento da informação auditiva no sistema nervoso central, demonstrada por um desempenho pobre nas habilidades e processos auditivos, entre eles a integração binaural, figura fundo e a resolução temporal (13).

O teste padrão de integração binaural é o teste de escuta dicótica, no qual é requerido ao ouvinte repetir todas as informações apresentadas em ambas as orelhas $(14,15)$. O uso de dígitos no teste de escuta dicótica minimiza a carga linguística imposta (16).

O Teste de Fusão Auditiva-Revisado (AFT-R) é utilizado para avaliar a habilidade de resolução temporal do processamento auditivo. Resolução temporal, particularmente na população pediátrica, é uma habilidade auditiva crítica necessária para um eficaz processamento auditivo. É definida como a capacidade do sistema auditivo para responder às mudanças rápidas de um estímulo sonoro (17).

Tais testes são propostos como triagem comportamental de processamento auditivo (16) e, descritos e recomendados, como presentes em uma bateria mínima de teste de processamento auditivo $(13,16)$.

Considerando que sujeitos com fissura labiopalatina possuem probabilidade maior de apresentar baixo peso (18), e que este é um indicador de extrema ligação com o desenvolvimento auditivo central, julgou-se necessário realizar um estudo retrospectivo comparativo dos resultados nos testes auditivos centrais de crianças com esta malformação craniofacial nascidas com baixo peso, visando auxiliar na caracterização de seu perfil audiológico e verificação da necessidade de encaminhamento para avaliação completa de processamento auditivo.

O objetivo deste estudo foi verificar e comparar o resultado de testes de processamento auditivo (o TDD e o AFT-R) de crianças portadoras de fissura labiopalatina com e sem baixo peso ao nascimento, com o de crianças sem esta malformação craniofacial e nascida com peso normal.

\section{MÉTODO}

Após aprovação pelo Comitê de Ética em Pesquisa em Seres Humano (Processo No 095/2009), foi realizado estudo retrospectivo e comparativo, por meio de análise de 73 prontuários de crianças submetidas à triagem do processamento auditivo no período de julho de 2004 a junho 2007, em um hospital do interior paulista especializado no atendimento de malformação craniofacial. Esta pesquisa foi realizada no ano de 2009.

Ser portador ou não de fissura labiopalatina, apresentar diagnóstico de audição periférica normal, se encontrar na faixa etária entre 7 e 12 anos de idade no momento da realização da triagem do processamento auditivo, ser de sexo masculino ou feminino e ter assinado o Termo de Consentimento Livre e Esclarecido foram os critérios de inclusão neste estudo. Foram excluídas crianças com fissura labiopalatina não operada, qualquer outra malformação associada presente, bem como com presença de perda auditiva diagnosticada.

Dos prontuários foram verificados os dados referentes ao sexo, peso ao nascimento, presença/ausência de fissura labiopalatina e o resultado de dois testes de processamento auditivo, o Teste Dicótico de Dígitos (TDD) e o Teste de Fusão Auditiva-Revisado (AFT-R), que são utilizados na triagem de processamento auditivo.

Com base nos dados coletados três grupos foram constituídos:

G1: 23 crianças com fissura labiopalatina e baixo peso ao nascimento;

G2: 25 crianças com fissura labiopalatina e peso normal ao nascimento;

G3: 25 crianças sem fissura labiopalatina e peso normal ao nascimento.

A Tabela 1 mostra a distribuição das crianças, quanto à idade e sexo nos três grupos amostrados.

Os pesos ao nascimento seguiram a seguinte classificação: baixo peso (1500 a 2500g); peso normal (2500 a 4000g) e macrossômico (acima de 4000g) (19).

Nos testes de processamento auditivo realizados (Teste de Fusão Auditiva AFT-R (20) e o teste Dicótico de Dígitos (21)) foi utilizado o audiômetro de dois canais SD50, acoplado a um CD player, a 50 dBNS.

No Teste de Fusão Auditiva-Revisado (AFT-R) foram apresentados tons puros com uma intensidade de 50 dBNS nas frequências de 250, 500, 1000, 2000 e $4000 \mathrm{~Hz}$, com um breve intervalo de tempo entre os pares de tons. 
Tabela I. Distribuição das crianças quanto ao Grupo, Idade eSexo.

\begin{tabular}{|c|c|c|c|c|c|c|c|c|c|c|c|c|c|}
\hline \multirow{2}{*}{ Grupo } & \multicolumn{12}{|c|}{ Idade (anos) } & \multirow{3}{*}{ Total } \\
\hline & 7 & & $\varepsilon$ & & 9 & & 10 & & I & & 12 & & \\
\hline & $M$ & $F$ & $M$ & $F$ & $M$ & $\mathrm{~F}$ & $M$ & $F$ & $M$ & F & $M$ & $\mathrm{~F}$ & \\
\hline GI & 0 & 2 & 2 & 1 & 5 & 0 & 7 & 1 & 0 & 2 & 3 & 0 & 23 \\
\hline G2 & | & 3 & 3 & 3 & 2 & 3 & 0 & 5 & 3 & 0 & 2 & 0 & 25 \\
\hline G3 & 4 & 3 & I & I & 2 & 2 & 5 & 2 & 2 & 0 & 0 & 3 & 25 \\
\hline Total & 5 & 8 & 6 & 5 & 9 & 5 & 12 & 8 & 5 & 2 & 5 & 3 & 73 \\
\hline
\end{tabular}

Legenda: $\mathrm{G} 1$ = 23 crianças com fissura labiopalatina e baixo peso ao nascimento; $\mathrm{G} 2=25$ crianças com fissura labiopalatina e peso normal ao nascimento; G3 = 25 crianças sem fissura labiopalatina e peso normal ao nascimento; $\mathrm{M}=$ masculino; $\mathrm{F}=$ feminino.

A criança deveria dizer se ouviu um ou dois tons. Para as crianças de sete anos é esperado um limiar de fusão de $9 \mathrm{~ms}$ e a partir de oito anos o esperado para o limiar de fusãoé de $8 \mathrm{~ms}$ (10).

No teste Dicótico de Dígitos (TDD) foi utilizada a versão gravada na etapa de Integração binaural, que consistiu em apresentar 40 pares de dígitos dissílabos diferentes, apresentados em dois pares de números simultaneamente a ambas as orelhas. A criança deveria repetir os quatro números ouvidos.

Neste estudo o TDD foi considerado alterado quando o resultado de qualquer uma das orelhas (direita ou esquerda) apresentou escore abaixo do esperado. Para as idades de 7 e 8 anos, é esperado uma porcentagem igual ou superior a $85 \%$ de acertos à direita e igual ou superior a 82\% à esquerda; para as idades 9 a 12 anos, é esperado escores iguais ou superiores a 95\% (10).

Por meio das informações citadas, foi realizada uma análise comparativa para cada grupo (sexo e idade) e entre os grupos. No entanto, nem toda a população amostrada realizou todos os dois testes descritos na metodologia devido às exigências do teste e as dificuldades apresentadas pela criança.

Os dados obtidos foram organizados em tabelas para facilidade de análise, comparação e apresentação seguindo os objetivos propostos. Os dados de Dicótico de Dígitos passaram pelo teste de normalidade de KolmogorovSmirnov e para comparação entre Grupos, Sexos e Idades foram realizados Análise de Covariância utilizando como covariável a Idade. Os dados de AFT-R não passaram pelo critério de normalidade de Kolmogorov-Smirnov, por isto utilizou-se o teste não paramétrico de Kruskal-Wallis para a comparação entre Grupos, Sexos e Idades. Em todos
Tabela 2. Valores médios e desvios-padrão do teste Dicótico de Dígitos dos diferentes grupos (GI, G2, G3) de acordo com as idades.

\begin{tabular}{lcccccc}
\hline Idade & \multicolumn{2}{c}{$\mathrm{Gl}$} & \multicolumn{2}{c}{$\mathrm{G} 2$} & \multicolumn{2}{c}{$\mathrm{G} 3$} \\
(anos) & Média & dp & Média & dp & Média & dp \\
\hline 7 & 84,7 & 6,2 & 70,5 & 16,0 & 88,1 & 9,3 \\
8 & 85,2 & 6,9 & 88,6 & 5,8 & 89,6 & 13,3 \\
9 & 86,0 & 20,7 & 92,3 & 4,4 & 97,8 & 2,6 \\
10 & 90,8 & 9,2 & 94,3 & 5,5 & 96,4 & 2,5 \\
11 & 94,7 & 5,7 & 96,7 & 3,4 & 99,1 & 1,9 \\
12 & 92,3 & 9,3 & 97,2 & 1,6 & 98,1 & 1,7 \\
\hline Total & 89,0 & 12,0 & 89,2 & 11,5 & 94,2 & 7,5 \\
\hline
\end{tabular}

Legenda: $\mathrm{G} 1=23$ crianças com fissura labiopalatina e baixo peso ao nascimento; $\mathrm{G} 2=25$ crianças com fissura labiopalatina e peso normal ao nascimento; G3 $=25$ crianças sem fissura labiopalatina e peso normal ao nascimento; $\mathrm{dp}=$ desviopadrão.

Tabela 3. Valores médios e desvios-padrão do teste AFT-R dos diferentes grupos (GI, G2, G3) de acordo com as idades.

\begin{tabular}{lcccccc}
\hline Idade & \multicolumn{2}{c}{$G$ G } & \multicolumn{2}{c}{$G 2$} & \multicolumn{2}{c}{$G 3$} \\
(anos) & Média & $d p$ & Média & $d p$ & Média & $d p$ \\
\hline 7 & 9,4 & 0,0 & 100,4 & 24,2 & 45,1 & 60,8 \\
8 & 84,4 & 65,1 & 59,4 & 48,6 & 11,9 & 9,1 \\
9 & 27,9 & 44,8 & 115,0 & 0,0 & 5,4 & 1,9 \\
10 & 50,6 & 52,5 & 29,9 & 46,2 & 6,4 & 9,2 \\
11 & 53,6 & 63,2 & 37,2 & 46,5 & 3,4 & 0,2 \\
I2 & 49,4 & 66,8 & 5,5 & 0,0 & 8,6 & 5,6 \\
\hline Total & 49,1 & 50,8 & 55,3 & 47,2 & 15,2 & 29,3 \\
\hline
\end{tabular}

Legenda: $\mathrm{G} 1=23$ crianças com fissura labiopalatina e baixo peso ao nascimento; $\mathrm{G} 2=25$ crianças com fissura labiopalatina e peso normal ao nascimento; G3 $=25$ crianças sem fissura labiopalatina e peso normal ao nascimento; $\mathrm{dp}=$ desviopadrão.

testes estatísticos adotou-se nível de significância de 5\% $(\mathrm{p}<0,05)$.

\section{RESULTADOS}

Para o teste Dicótico de Dígitos (Tabela 2) a Análise de Covariância não mostrou haver diferença estatisticamente significante entre os grupos $(p=0,340)$, entre os sexos $(p=0,985)$ e nem para a interação entre estes fatores $(\mathrm{p}=0,112)$, porém a co-variável Idade mostrou relação estatisticamente significante com o Dicótico de Dígitos $(\mathrm{p}<0,001)$. O coeficiente de correlação da idade com o Dicótico de Dígitos foi r $=0,48$.

Para o AFT-R (Tabela 3) o teste de Kruskal- Wallis não mostrou haver diferença estatisticamente significante entre os grupos $(p=0,136)$, entre os sexos $(p=0,371)$ e nem entre as idades $(p=0,378)$. 


\section{DISCUSSÃO}

Os escores alterados tanto no AFT-R, quanto no teste Dicótico de Dígitos demonstrado pelas crianças com fissura labiopalatina nascidas com baixo peso (Tabela $2 \mathrm{e}$ Tabela 3) sugere uma habilidade de resolução temporal fraca, assim como, dificuldade com a integração binaural e figura-fundo para sons verbais. Este resultado leva-se a pensar que tais crianças podem manifestar dificuldade de distinguir e identificar rapidamente os sons apresentados no discurso, além de que, um pobre desempenho na integração binaural e figura-fundo pode ser expresso nos sintomas comportamentais de dificuldade em ruído de fundo, ou dificuldade em ouvir duas conversas ao mesmo tempo $(14,15,22)$.

As alterações encontradas nos testes propostos deste estudo podem ser ainda justificadas pela capacidade reduzida de relembrar a informação auditiva $(10,14)$, capacidade esta requisitada nos testes de AFT-R e Dicótico de Dígitos.

Estudo (11) visando verificar o desempenho de crianças com fissura labiopalatina no teste Dicótico de Dígitos e compará-los aos de um grupo sem fissura labiopalatina, relata diferença no desempenho de meninos e meninas, no qual as meninas apresentaram índices de acerto inferiores às do grupo controle estudado, diferindo do presente trabalho, que se mostrou sem significância entre os sexos. Consideração deve ser feita, uma vez que a etapa estudada pelos autores (11) enfocou etapa de escuta direcionada, na qual é requerida a separação binaural, em que o ouvinte presta atenção para um sinal apresentado em uma orelha, enquanto ignora o outro sinal apresentado simultaneamente na outra orelha. Isto tem sido referido como atenção auditiva direcionada (23). Contrastando, no presente estudo foi requerida a integração binaural, em que o ouvinte deve repetir todas as informações apresentadas simultaneamente em ambas as orelhas $(14,15)$, referida algumas vezes como atenção auditiva dividida (23). Salienta-se que diferentes tarefas dicóticas revelam diferentes resultados (11).

Pesquisadores (10) demonstraram uma alta porcentagem de crianças com fissura isolada de palato com seus piores desempenhos no AFT-R (95\%) e no TDD (95\%).

Alteração no AFT-R na população com fissura labiopalatina também foi verificado por outros pesquisadores (9), que estudaram os limiares médios de fusão auditiva de crianças com e sem fissura labiopalatina entre 6 e 7 anos, demonstrando que em crianças que apresentaram esta malformação craniofacial, os limiares de fusão auditiva foram significantemente mais altos quando comparados ao grupo sem fissura labiopalatina, sendo considerados sugestivos de alteração do processamento auditivo temporal. Os autores atribuíram à privação sensorial decorrente das otites médias apresentadas nos primeiro anos de vida. Este trabalho não deu ênfase ao estudo de histórias de otites médias na população estudada.

Pesquisadores (24) apontam uma melhora em tarefas de resolução temporal com o aumento da idade, o presente estudo não encontrou diferença estatisticamente significante entre as idades avaliadas. Similar resultado foi obtido em pesquisa (17) com crianças entre 7 e 18 anos de idade, utilizando um o teste de resolução temporal GIN (Gaps-In-Noise) (25).

A significância da variável idade no Teste Dicótico de Dígitos evidenciada neste estudo corrobora com a literatura, afirmando que a melhora da das habilidades auditivas com o aumento da idade, especialmente entre os oito e dez anos, está associada ao processo de maturação (26). Deve-se ressaltar a importância da maturação do sistema auditivo da criança. Os testes de processamento auditivos são dependentes da função neural, portanto, os resultados devem ser analisados enfatizando a neuromaturação $(15,23)$.

\section{CONCLUSÃO}

Os resultados do presente trabalho demonstram que crianças com fissura labiopalatina e baixo peso ao nascer, embora sem significância estatística, apresentam maiores alterações nos testes de processamento auditivo utilizados ao comparar com crianças com fissura labiopalatina isolada e com crianças sem esta malformação craniofacial e sem baixo peso ao nascer. Ressalta-se ainda que o aumento da idade melhorou o desempenho no teste Dicótico de Dígitos.

\section{REFERÊNCIAS BIBLIOGRÁFICAS}

1. Silva DPC, Martins RHG. Análise das emissões otoacústicas transientes e dos potenciais evocados auditivos do tronco encefálico em neonatos com hiperbilirrubinemia. Braz J Otorhinolaryngol. 2009, 75(3):381-6.

2. Yin R, Wilkinson AR, Chen C, Brosi DM, Jiang ZD. No close correlation between brainstem auditory function and peripheral auditory threshold in preterm infants at term age. Clin Neurophysiol. 2008, 119(4):791-5.

3. Pereira PKS, Martins AS, Vieira MR, Azevedo MF. Programa de triagem auditiva neonatal: associação entre perda auditiva e fatores de risco. Pró-Fono. 2007, 19(3):267-78. 
4. Lamônica DAC, Carlino FC, Alvarenga KF. Avaliação da função auditiva receptiva, expressiva e visual em crianças prematuras. Pró-Fono. 2010, 22(1):19-24.

5. Cristobal R; Oghalai JS. Hearing loss in children with very low birth weight: current review of epidemiology and pathophysiology. Arch Dis Child Fetal Neonatal. 2008, 93:462-68.

6. Pegoraro-Krook MI, Dutka-Souza JCR, Magalhães LCT, Feniman MR. Intervenção Fonoaudiológica na Fissura Palatina. In: Ferreira LP, Befi-Lopes DM, Limongi SCOTratado de Fonoaudiologia. São Paulo: Roca; 2004. p. 447-50.

7. D'MelloJ, Kumar S. Audiological findings in cleft palate patients attending speech camp. In: Indian J Med Res. 2007, 125:777-82.

8. Amaral MIR, Martins JE, Santos MFC. Estudo da audição em crianças com fissura labiopalatina não-sindrômica. Braz J Otorhinolaryngol. 2010, 76(2):164-71.

9. Cassab TV, Zorzetto NL. Teste da fusão auditiva-revisado (AFT-R) em crianças com fissura Labiopalatina. ACTA ORL/ Técnicas em Otorrinolaringologia. 2006, 24(4):272-6.

10. Boscariol M, André KD, Feniman MR. Crianças com fissura isolada de palato: desempenho nos testes de processamento auditivo. Braz J Otorhinolaryngol. 2009, 75(2):213-20.

11. Lemos ICC, Monteiro CZ, Camargo RA, Rissato, ACS, Feniman, MR. Teste Dicótico de Dígitos (etapa de escuta direcionada) em crianças com fissura labiopalatina. Rev Bras Otorrinolaringol. 2008, 74(5):662-7.

12. Duarte TF, Salvador KK, Cruz MS, Feniman MR. Desempenho de Crianças com Fissura Labiopalatina na Avaliação do Processamento Auditivo. In: Anais da XVI Jornada Fonoaudiológica de Bauru "Prof ${ }^{\mathrm{a}} \mathrm{Dr}^{\mathrm{a}}$ Katia deFreitas Alvarenga"; 2009, 26-29 agosto; Bauru, Brasil. Bauru. Universidade de São Paulo - Faculdade de Odontologia de Bauru; 2009. p. 60.

13. American Speech-Language-Hearing Association. (2005) (Central) Auditory Processing Disorders [Technical Report]. In: http://www.asha.org/policy.

14. Bellis TJ. Assessment and management of central auditory processing disorders in the educational setting. From Science to practice. ( $2^{\text {nd }}$ Edition) San Diego: Singular, 2003.

15. Guenette LA. How to administer the Dichotic Digit Test. Hear J. 2006, 59(2):50.
16. Jerger, J, Musiek, F. Report of the Consensus Conference in the diagnosis of auditory processing disorders in schoolaged children. J Am Acad Audiol. 2000, 11:467-74.

17. Shinn JB, Chermak GD, Musiek FE. GIN (Gaps-In-Noise) performance in the pediatric population. J Am Acad Audiol. 2009, 20:229-38.

18. Wyszynski DF, Sarkozi A, Vargha P, Czeizel AE. Birth weight and gestational age of newborns with cleft lip with or without cleft palate and with isolated cleft palate. J Clin Pediatr Dent. 2003, 27(2):185-90.

19. Fernández-Sanabria RV. Valor de Hemoglobina em la gestante y su relación com el parto pretérmino y peso del recién nacido en pacientes atendidas en el Hospital Santa Rosa durante el periodo Abril 2001- Octubre 2001. .[Dissertação]. Lima(Peru): Universidad del Peru-Facultad de Medicina. 2002.

20. McCrosckey R, Keith RW. AFT-R: Auditory Fusion TestRevised. San Antonio, TX: Psychological Corporation; 1996.

21. Santos MFC, Pereira LD. Escuta com Dígitos. In: Processamento Auditivo Central Manual de Avaliação. São Paulo: Lovise. 1997; p.147-49.

22. Cameron S, Dillon H. Auditory Processing Disorder from Screening to Diagnosis and Management - A Step-byStep Guide-Audiology Now. (Winter 2005 edition), p. 4755.

23. Bellis TJ. Interpretation of APD tests results. In: Parthasarathy TK, editor. An introduction to auditory processing disorders in children. Mahwah:Lawrence Erlbaum. 2006. p.245-60.

24. Hautus MJ, Setchell GJ, Waldie KE, Kirk IJ. Age-related improvements in auditory temporal resolution in readingimpaired children. Dyslexia. 2003, 9:37-45.

25. Musiek F, Shinn J, Jirsa B, Bamiou D, Baran J, Zaidan E. GIN (Gaps-In-Noise) test performance in subjects with confirmed central auditory nervous system involvement. Ear Hear. 2005, 26:608-18.

26. Neves IF, Schochat E. Maturação do processamento auditivo em crianças com e sem dificuldades escolares. PróFono. 2005, 17(3):311-20. 